\title{
Evaluating Scientific Domain Ontologies FOR THE ELECTROMAGNETIC KNOWLEDGE DOMAIN: A GENERAL METHODOLOGY
}

\author{
Alessandra Esposito ${ }^{1}$, Marco Zappatore ${ }^{1}$ and Luciano Tarricone ${ }^{1}$ \\ ${ }^{1}$ Department of Engineering for Innovation, University of Salento, \\ via per Monteroni, 73100 Lecce - Italy \\ \{alessandra.esposito; marcozappatore; luciano.tarricone\}@unisalento.it
}

\begin{abstract}
The adoption of ontologies as a formalized approach to information codification is a constant-growing phenomenon in scientific research. Moreover, knowledge sharing and reuse can be improved by adopting hierarchical and modular frameworks and therein embedding available ontologies. Unfortunately, merging procedures may bring about severe, time-consuming problems if a careful selection process is not carried out. Based on these considerations, we propose a methodology for evaluating and selecting higher-level ontologies, given the lower-level ones. Our proposal is based on the computation of ad-hoc metrics which take into account structural and semantic aspects and on the adoption of a multi-decisional analysis procedure. The methodology has been applied to identify ontologies suitable for providing a scientific domain high-level codification to Electromagnetism. Two well-known scientific domain ontologies have been selected and evaluated with the proposed methodology.
\end{abstract}

\section{KEYWORDS}

Ontology, Metric Computation, Multi-Criteria Decisional Analysis, Electromagnetic Knowledge Domain.

\section{INTRODUCTION}

Nowadays many scientific domains benefit from ontology codification [1]. Healthcare [2], Biology [3], Astronomy [4] and Geology [5] are only a few examples. Other ontologies provide formalizations on more generic and transversal areas, such as Math and Physics [6-9]. Electromagnetic (EM) domain codification, instead, is still at a germinal phase. Only few, specific EM subdomains have been considered until now, such as Remote Sensing [10].

In order to fill this gap, a first tentative for defining a global ontological infrastructure for the entire EM knowledge domain was proposed by the authors in OntoCEM (Ontological Codification of ElectroMagnetism) [11]. Such framework was modeled as a stack of proprietary and third-party ontological modules organized according to different levels of semantic abstraction. Ontologies gathering general scientific concepts, generally known as domain ontologies, fill in the higher layers whilst more specific EM-related concepts (sub-domain and application level ontologies) occupy the lower levels. The adopted architecture is a widely accepted technique to increase the potential reusability of semantic frameworks [12].

In order to choose the ontologies best-suited for providing a higher-level to OntoCEM, we adopted a methodology based on the computation of a set of ad-hoc metrics and on their elaboration in a multi-decisional analysis technique. Our metrics evaluate ontologies from different points of view, such as structural aspects, semantic completeness, domain adequacy, DOI : 10.5121/ijwest.2011.2301 
International Journal of Web \& Semantic Technology (IJWesT) Vol.2, No.3, July 2011

reusability and integrability. The multi-decisional analysis technique, instead, is used to calculate global synthetic evaluation indexes upon which the ontologies are ranked. Even though our work was inspired by the need of comparing scientific domain ontologies, the procedure is general and applicable to other situations. Therefore the analysis of scientific domain ontologies, which is considered throughout the whole paper, can be regarded as a concrete evaluation case study of a general-purpose methodology.

The paper is structured as follows. Section 2 briefly reviews other works about ontology evaluation. Section 3 details OntoCEM structure and contents. Section 4 describes the proposed approach. Section 5 presents candidate ontologies. Section 6 defines a set of metrics and their calculations over the candidate ontologies. Section 7 describes the adopted overall scoring technique.

\section{RELATED WORK}

Integration procedures among ontologies belonging to different semantic layers has many facets [13] and may require carrying out critical tasks, such as adaptation, deletion or relocation of knowledge, which render onerous the merging procedure. In order to reduce the impact of these problems on ontology realization and maintenance, a careful analysis and selection among available ontologies is highly desirable. In order to render the evaluation technique rigorous, metric computation is desirable as well. Metrics measuring different ontological aspects have been adopted since late '90s [16] taking inspiration from software engineering classification methodologies. Metrics based on both the analysis of the adopted design techniques [17] and the codified contents [18] are available and widely used as well. The former consider size and structural aspects by using automated or semi-automated tools and do not take into account a qualitative analysis of the entities codified into the knowledge base. The latter try to analyze the semantic meaning of the ontology contents and quite often require a domain expert contribution. Other works tackle ontology matching issues by examining semantic relationships of words in WordNet [33]. In [34], lexical similarities (i.e., hypernymy, hyponymy, etc.) are considered whilst in MOMIS (Mediator Environment for Multiple Information Source) [35] a lexical matrix is created by analyzing WordNet synonym sets.

Our work proposes a systematic approach dealing with both aspects. It is based on the computation of different kinds of metrics and joins them up by using a consolidated ranking procedure. The proposed metrics are simple and effective. They take into account different points of view: structural, semantic and procedural. The former require a simple and global high-level inspection of the ontology and can be automated. Semantic metrics count on domain expert mediation. Procedural metrics are directly devoted to the estimation of integration effort and reusability, by carrying out a concrete simulation of integration activities [29] [30]. The proposed metrics are used as input of a multi-criteria decision analysis method. In this way, global synthetic evaluation indexes are calculated, thus coping with criteria giving discordant evaluations. Finally, the entire evaluation process has been adopted in a real-life application, which inspired the overall proposal, i.e. the choice of the best-suited ontology for providing a scientific mid-level to our OntoCEM framework. Two important publicly available ontologies, i.e. the ontologies published by the Astronomical Department of the University of Maryland (UMD) [21] and the Semantic Web for Earth and Environmental Terminology (SWEET) Ontologies [22], are compared for this purpose. At our knowledge, this is the first work in which a thorough evaluation methodology based on heterogeneous metric computation is applied on such relevant ontologies. Large ontology evaluation is usually coped with automatic computation of size metrics only. On the other side, subjective semantic metrics are generally computed only against small, example ontology. 


\section{ONTOCEM OVERVIEW}

According to a well-known and widely adopted model [25], OntoCEM is proposed as a stack of semantic layers with different degrees of abstraction (see Fig. 1).

In such a structure, domain and subdomain ontologies are placed on top of the stack whilst more specific and application-related ontologies are located in lower levels.

Ontologies codifying concepts strictly related to EM populate the two lower layers of the stack. Ten EM domain ontologies collect semantic higher-level concepts describing EM fields and waves, antennas, EM measurements, EM propagation mechanisms, etc. The very bottom layer, instead, gathers modules describing EM applications, such as antenna CAD techniques, EM numerical analysis methods, EM shielding procedures, EM radio propagation models, etc.

OntoCEM ontologies have been codified in OWL2-DL language [14] and will be made progressively available [15].

On top of proprietary ontologies, an upper level has been added as a third layer, in order to host the ontologies codifying general and abstract physical and mathematical entities, such as units of measurement, material properties, etc. These ontologies were taken from publicly available frameworks, in order to improve shareability and reusability.

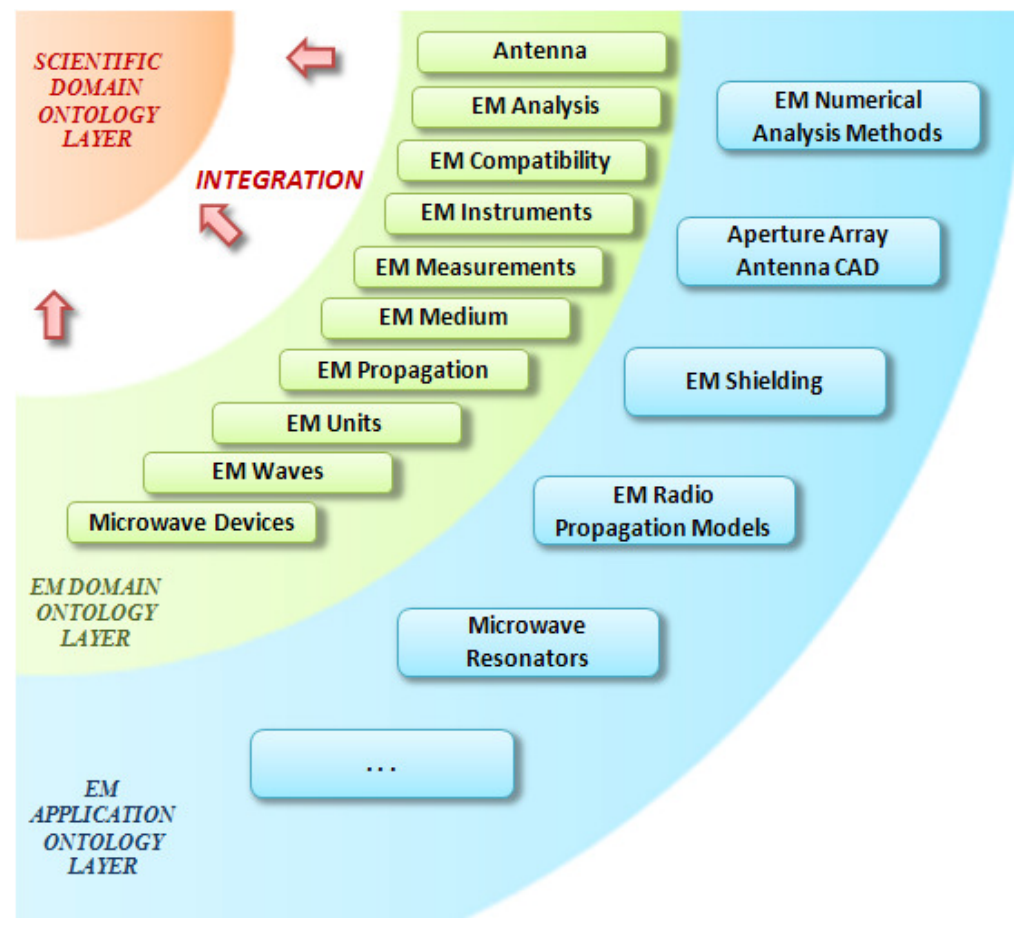

Figure 1. OntoCEM architecture 
International Journal of Web \& Semantic Technology (IJWesT) Vol.2, No.3, July 2011

\section{The Evaluation Methodology}

In order to choose the best suited set of ontologies for the scientific domain layer, we adopted a possibly rigorous evaluation procedure, depicted in Figure 2 and described below.

First of all, candidate ontologies have to be selected. We identified a pool of mandatory requirements that must be fulfilled during this phase.

- Semantic coverage: ontologies gathering a large amount of scientific entities are to be preferred. In this way, the reusability of the acquired knowledge towards different directions (i.e. towards different application level ontologies) is maximized.

- Open availability: selected ontologies must be publicly available, so that both free access and local modifications to their contents are viable.

- Codification language: codification in OWL-DL [14] format is preferred.

- Modularity: the modularity of the candidate sets represented an important additional value, as it supports selective imports into proprietary ontologies.

After the selection of candidate ontologies, we evaluate them by computing a set of metrics. Ontology evaluation is a multi-feature analysis, based on the calculation of heterogeneous metrics, dealing with different design and domain aspects. Therefore, we propose three groups of metrics.

- Size and structural metrics: quantify basic design aspects such as modularity and the amount of codified entities. These metrics do not depend from low-level ontologies and their computation can be automated.

- Content metrics: evaluate how the contents of the candidate ontologies are organized and their suitability to be integrated with low-level ontologies. These metrics are subjective, i.e. they depend on the opinion of the expert of the knowledge domain.

- Integration metrics: measure features that are relevant in the merging procedure such as the number of suitable semantic ancestors for low-level concepts and the probability of reuse for properties. These metrics are used to weigh the integration effort and ontology reusability.

The metrics listed above monitor heterogeneous features and may produces discordant results. Therefore, as third step, a global methodology to rank the ontologies in a coherent way is adopted: based on a multi-attribute decision making procedure, the domain-level ontology is finally chosen.

In the following sections we provide further details on each task, by describing how this methodology was applied to identify the domain-level ontology for OntoCEM. 


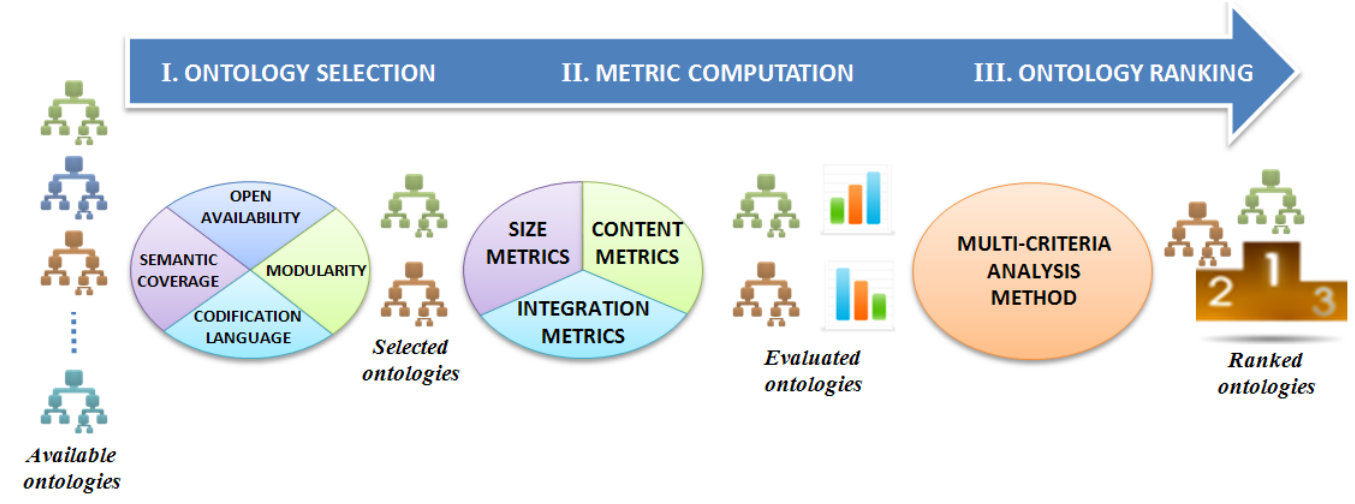

Figure 2. The evaluation procedure

\section{Candidate Ontology Selection}

As a first step, a selection based on high-level qualitative considerations was carried out. On the basis of criteria listed in the previous section, we identified two candidates: the ontologies published by the Astronomical Department of the University of Maryland (UMD) [21], part of the IVOAT project (International Virtual Observatory Alliance Thesaurus) [26] and the Semantic Web for Earth and Environmental Terminology (SWEET) Ontologies [22], developed by NASA JPL [27]. Both sets of ontologies provide great corpora of scientific concepts and properties, thus being amenable to be used as upper-level ontologies for the EM subdomain. They are codified in the OWL-DL format and are publicly available. Finally, both frameworks feature a modular architecture.

UMD ontologies are centered around a single empty ontology ("IVOAO_root") which gathers remaining modules, without any further hierarchy. Module repartition follows a pragmatic domain-oriented strategy [31], according to which the "Astronomy" module collects astronomical concepts, the "Physics" and "Physical Theory" modules comprise all the available terms related to Physics, including acoustics, electromagnetism, mechanics, dynamics, thermodynamics, etc.

SWEET ontologies, instead, are structured around eight top-level ontologies/concepts, which subsume remaining modules. The concepts are partitioned into orthogonal dimensions (facets) [32] according to reductionism guiding principles. For instance: "Matter" subsumes entities describing material things (from chemical elements to industrial products); "Process" subsumes all kinds of available processes (spatial, temporal, chemical, physical) and so on.

\section{Metric Computation}

As detailed in Section 3, our metrics are used as ranking criteria in a multi-decisional analysis procedure. Therefore, they must share the same numerical scale and ranges (normalized values). We chose the [0;1] range as reference for all the proposed metrics. Out-of-range scores have been normalized without introducing neither distortions nor biases with the following linear scaling transformation (1):

$$
x_{\text {norm }}=\frac{x_{a-} \min _{i}\left\{x_{i}\right\}}{\max _{i}\left\{x_{i}\right\}-\min _{i}\left\{x_{i}\right\}}
$$


where $x_{a}$ is the actual variable, $x_{n o r m}$ its normalization and $x_{i}$ all its possible values. Moreover, the overall problem must be configured as a maximization (minimization) one. Therefore, when needed, metrics are suitably converted.

The following subsections are devoted to each group we partitioned our metrics into: 1) size and structural; 2) content-related; 3) integration-related.

\subsection{Size and Structure Metrics}

\section{Class to Entity ratio (CtEr)}

It computes the ratio between the total number of classes $(\mathrm{nCl})$ and the total number of entities $(n E n t)$. Therefore it weights how strong the taxonomical component is.

$$
C t E r=\frac{n C l}{n E n t}
$$

\section{Property to Entity ratio (PtEr) and Instance to Entity ratio (ItEr)}

They quantify respectively property and instance presence, as it is recognized to enrich the ontology [24]. Indeed, the less properties and instances there are, the more the ontology resembles a mere taxonomy made up only of "is- $A$ " relationships. Their formulas are the following:

$$
\begin{gathered}
P t E r=\frac{n O P+n D P}{n E n t} \\
I t E r=\frac{n I n s t}{n E n t}
\end{gathered}
$$

where $n O P, n D P$ and $n I n s t$ represent the number of Object Properties, Datatype Properties and Instances respectively.

\section{Entities per Module ratio (EpMr)}

It is the ratio between the number of entities (nEnt) and the number of modules $(n M)$.

$$
E p M r=\frac{n E n t}{n M}
$$

This is a non-normalized, bounded quantity. Its lower bound is the case of one entity per module, $E p M r_{\text {min }}=1$, and its upper bound is represented by all the entities defined in only one module, that gives: $E p M r_{M A X}=n E n t$. Therefore the normalized ratio $\left(E p M r_{n o r m}\right)$ is:

$$
E p M r_{\text {norm }}=\frac{\left(\frac{n E n t}{n M}\right)-1}{n E n t-1}
$$

In order to avoid problematic concept localization procedures, the value of this metrics should be as low as possible. Therefore, the complemented metrics (7) is considered:

$$
E p M r=1-E p M r_{\text {norm }}
$$


International Journal of Web \& Semantic Technology (IJWesT) Vol.2, No.3, July 2011

Table I compares the candidates in terms of the metrics defined above and highlights the best value for each of them.

Table 1. Size metrics for candidate ontologies

\begin{tabular}{|c|c|c|}
\hline & UMD & SWEET \\
\hline Classes & 4238 & 4354 \\
\hline Object Properties & 221 & 540 \\
\hline Datatype Properties & 92 & 39 \\
\hline Instances & 268 & 1935 \\
\hline Total & 4819 & 6868 \\
\hline Modules & 13 & 196 \\
\hline CtEr & 0.88 & 0.63 \\
\hline PtEr & 0.06 & 0.08 \\
\hline ItEr & 0.05 & 0.28 \\
\hline EpMr & 0.923 & 0.995 \\
\hline
\end{tabular}

UMD ontologies are preferable in terms of $C t E r$, whilst SWEET ontologies show better values for the other metrics.

\subsection{Content Metrics}

As size metrics do not convey any domain-related information, we propose also metrics based on the evaluation of contents belonging to candidate ontologies against EM subdomain ontology contents. In order to compute such metrics, we partitioned the entities available in candidate ontologies into the following three categories.

Scientific (S) entities are scientific concepts capable of acting as valid superclasses or reusable properties for EM concepts defined in OntoCEM proprietary ontologies. $S$ entities can provide useful information being inherited by EM concepts. Their number is indicated as $n S$.

Electromagnetic $(\boldsymbol{E})$ entities are concepts concerning EM which have been defined in the candidate ontologies. As EM concepts are located in OntoCEM modules as well, $E$ entities may reveal the occurrence of semantic conflicts during the integration procedure. Therefore it is preferable to have only a small quantity $(n E)$ of them in candidate ontologies.

Unusable $(\boldsymbol{U})$ entities are entities that belong neither to $S$ nor to $E$ set. The number $n U$ of $U$ entities should be as low as possible, in order to reduce ontology loading times. We indicate as ES modules the modules comprising at least one $S$ or $E$ entity.

Table 2 presents some $S$ and $E$ entities. 
International Journal of Web \& Semantic Technology (IJWesT) Vol.2, No.3, July 2011

Table 2. Examples of $S$ and $E$ entities

\begin{tabular}{|c|c|}
\hline Scientific $(S)$ Entities & Electromagnetic (E) Entities \\
\hline \multicolumn{2}{|c|}{ Classes } \\
\hline $\begin{array}{c}\text { Process; Physical Property; Energy; } \\
\text { Function; Scientific Model; Vector; } \\
\text { Integral; Transmitter; Orientation; Spatial } \\
\text { Distribution; Logarithmic Scale; } \\
\text { Algorithm... }\end{array}$ & $\begin{array}{l}\text { Remote Sensing; Magnetic Field; Electric } \\
\text { Dipole; EM Process; Diffraction; Wave } \\
\text { Propagation; EM Spectrum; Antenna; } \\
\text { Phase Velocity; Wavelength; Microwave... }\end{array}$ \\
\hline \multicolumn{2}{|c|}{ Object Properties } \\
\hline $\begin{array}{l}\text { has Effect; has Component; has Unit; has } \\
\text { Force; has Direction; has Derivative... }\end{array}$ & $\begin{array}{l}\text { radiate; polarize; has Wave Process; has } \\
\text { Frequency; has Spectral Band ... }\end{array}$ \\
\hline \multicolumn{2}{|c|}{ Datatype Properties } \\
\hline $\begin{array}{l}\text { has Probability; has Scale; has Numeric } \\
\text { Value; has Symbol; has Lower Limit... }\end{array}$ & $\begin{array}{l}\text { has Scattering Coefficient; has Relative } \\
\text { Permittivity; has Resonant Frequency... }\end{array}$ \\
\hline \multicolumn{2}{|c|}{ Instances } \\
\hline $\begin{array}{l}\text { Al; Sn; X Axis; Meter; per Second; dB; } \\
\text { Joule; dimensionless Unit; FFT; radian... }\end{array}$ & $\begin{array}{l}\text { SNR; Ohm; Refractive Index; Megahertz; } \\
\text { Tesla; Siemens; Volt per meter... }\end{array}$ \\
\hline
\end{tabular}

On the basis of such notation, we propose three content metrics calculated on ES modules. These metrics require the contribution of a domain expert, responsible for locating $S$ and $E$ entities in the candidate ontologies.

\section{Domain Scientific Richness (DSR)}

This metric quantifies the presence of $S$ concepts.

$$
D S R=\frac{n S}{n S+n E+n U}
$$

\section{Domain EM Richness (DER)}

It quantifies the presence of $E$ concepts.

$$
D E R=1-\frac{n E}{n S+n E+n U}
$$

\section{Loading Overhead (LO)}

It quantifies the presence of $U$ concepts.

$$
L O=1-\frac{n U}{n S+n E+n U}
$$


International Journal of Web \& Semantic Technology (IJWesT) Vol.2, No.3, July 2011

Table 3 shows that UMD and SWEET content metrics have discordant values and highlights best values. This confirming the need of a unifying ranking methodology (see Section 7).

Table 3. Content metrics for candidate ontologies

\begin{tabular}{|c|c|c|}
\hline & UMD & SWEET \\
\hline $\mathbf{n S}$ & 1361 & 1900 \\
\hline $\mathbf{n E}$ & 182 & 225 \\
\hline $\mathbf{n U}$ & 1529 & 1054 \\
\hline Total & 3072 & 3179 \\
\hline ES Modules & 11 & 83 \\
\hline \hline DSR & 0.44 & 0.59 \\
\hline DER & 0.94 & 0.93 \\
\hline LO & 0.5 & 0.66 \\
\hline
\end{tabular}

\subsection{Integration Metrics}

In order to evaluate how suitable a candidate ontology is with respect to integration, we propose an approach which is based on the simulation of integration tasks.

First of all, a set of concepts (named benchmark entities, $B E$ ) codified in the lower-level ontology has to be identified. The simulation of integration tasks will be carried out on such entities, which are chosen on the basis of "structural" and/or "semantic" considerations. We selected classes subsuming a great number of concepts and/or relevant from a domain point of view. Table 4 enlists the chosen entities. They are taken from each of the EM domain modules depicted in Fig. 1.

Table 4. OntoCEM benchmark entities

\begin{tabular}{|c|l|}
\hline EM Concept & \multicolumn{1}{|c|}{ Role in OntoCEM } \\
\hline $\begin{array}{c}\text { OntoCEM module } \\
\text { Antenna }\end{array}$ & Root concept subsuming all antenna typologies \\
\hline Antenna & $\begin{array}{l}\text { Root concept describing scientific models that estimate } \\
\text { signal attenuation due to Path Loss in wireless } \\
\text { communication systems }\end{array}$ \\
\hline Radio Propagation Model \\
\hline EM Propagation & $\begin{array}{l}\text { Particular solution of Maxwell's equations with electric } \\
\text { field assuming the same magnitude and phase in all planes } \\
\text { perpendicular to the direction of propagation }\end{array}$ \\
\hline MilliVolt per meter & $\begin{array}{l}\text { A common unit of measurement for electric field strength } \\
\text { values Waves }\end{array}$ \\
\hline EM Units & Root concept extended by all other insulator media \\
\hline Dielectric Medium &
\end{tabular}


International Journal of Web \& Semantic Technology (IJWesT) Vol.2, No.3, July 2011

\begin{tabular}{|c|c|}
\hline EM Medium & \\
\hline EM Analysis & $\begin{array}{l}\text { It is a general numerical technique for solving EM problems } \\
\text { stated in terms of an inhomogeneous equation }\end{array}$ \\
\hline RF Measurement & $\begin{array}{l}\text { Root concept subsuming all kinds of measurements } \\
\text { performed at RF frequencies }\end{array}$ \\
\hline Plane Wave Shielding & $\begin{array}{l}\text { It is the process that determines a total or partial block of } \\
\text { EM radiation (propagating as a plane wave) in a far field } \\
\text { region }\end{array}$ \\
\hline $\begin{array}{c}\text { Spectrum Analyzer } \\
\text { EM Instruments }\end{array}$ & $\begin{array}{l}\text { Fundamental EM instrument for measuring the frequencies } \\
\text { present in a complex signal or resulting from modulation on } \\
\text { a carrier }\end{array}$ \\
\hline $\begin{array}{c}\text { Passive Microwave Device } \\
\text { Root concept subsuming all passive components operating } \\
\text { at microwave frequencies }\end{array}$ \\
\hline
\end{tabular}

Once benchmark entities have been selected, three metrics recalling and enhancing some metrics proposed by Zhang in [17] are computed.

\section{Inheritance Mismatch (IM)}

This metric was defined starting from the so-called Depth of Inheritance metric (DoI) [17]. DoI measures the distance of the class best suited to subsume the benchmark entity from the root class of the candidate ontology. It does not provide any information about the validity of the ancestors from a domain viewpoint. Instead, we propose to take into account the opinion expressed by a domain expert about the amenability of ancestors to be superclasses of the benchmark entity. Based on such evaluation, $I M$ computes the ratio between the overall number of "appropriate ancestors" ( $n S A)$ and DoI, according to the following formula:

$$
I M=\frac{\sum_{i=1}^{n B E} I M_{i}}{n B E}, \quad I M_{i}=\frac{n S A_{i}}{D o I_{i}}
$$

As $I M_{i}$ refers to the $i$-th benchmark entity, $I M$ is the arithmetic mean over the $n B E$ evaluated benchmark entities. The closer to zero $I M$ is, the more unfitting the superclasses are.

\section{Inheritance Deviation (ID)}

This metric is derived from $D o I$ as well. It takes into account the maintenance issues which could occur when the distance from the root class is too long (i.e. DoI is high). In this case, a modification in higher-level scientific domain concepts can involve relevant modifications in the lower-level EM ontologies [17]. Therefore we set a reference value $\left(D o I_{R E F)}\right.$ and measure the deviation of $D o I_{i}$ from it. In order to align $I D_{i}$ to other metrics, we normalized it to the range $[0 ; 1]$ and converted it to a maximization metric. $I D$ is the arithmetic mean among all $I D_{i}$.

$$
I D=\frac{\sum_{i=1}^{n B E} I D_{i}}{n B E}, \quad I D_{i}=1-\frac{\left|D o I_{R E F}-D o I_{i}\right|}{\max \left\{D_{i}, D_{R E F}\right\}}
$$


The closer to one $I D$ is, the closer to the reference the actual $D o I$ is. We selected $D o I_{R E F}=3$ as a proper value.

\section{Domain Property Reusability (DPR)}

It analyzes how the descriptive statements for the benchmark entity can be rendered. $D P R$ is the ratio between the reusable OWL properties belonging to higher-level ontologies $\left(n R P_{i}\right)$ and the number of natural language restrictions $\left(n N L R_{i} \geq n R P_{i}\right)$ needed to codify the descriptive statement for the $i$-th benchmark entity:

$$
D P R=\frac{\sum_{i=1}^{n B E} D P R_{i}}{n B E}, \quad D P R_{i}=\frac{n R P_{i}}{n N L R_{i}}
$$

The closer to one the DPR is, the more preferable the ontology is. Indeed, by counting the number of reusable properties, we measure how the higher-level ontology facilitates the integration enhancement. On the contrary, a $D P R$ close to zero denotes a small reusability.

Considering all the benchmark entities detailed in Table 4, the candidate ontologies feature the following mean values in terms of $I M, I D$ and $D P R$ metrics (see Table 5). Best values are highlighted.

Table 5. Integration metrics for candidate ontologies (arithmetic mean among all the benchmark entities)

\begin{tabular}{|c|c|c|}
\hline Metric & UMD & SWEET \\
\hline IM & 0.667 & 0.91 \\
\hline ID & 0.398 & 0.659 \\
\hline DPR & 0.386 & 0.711 \\
\hline
\end{tabular}

\subsection{Computing integration metrics on an OntoCEM benchmark entity}

Table 5 proposed the overall value for $I M, I D$ and $D P R$ metrics. In this section we provide a complete evaluation of those metrics on a sample benchmark entity: the Dielectric Medium benchmark class belonging to EM Medium ontology (see Table 4).

A dielectric medium is a particular material, classified according to its electrical properties, that behaves as an electric insulator [28]. This class directly subsumes 10 classes in OntoCEM, which codify dielectric materials with respect to their chemical structure and frequency behavior such as: Microwave Dielectric, Ceramic Dielectric, Low-Loss Dielectric, Air-Gap Dielectric, PET Film Dielectric, etc.

Neither UMD nor SWEET ontologies contain this entity, therefore we have only to search for appropriate ancestor classes (in the opposite case, a declaration of equivalence among classes would be needed). As shown in Fig. 3, the former offer, as superclass, the Matter entity, without any other ancestor. The latter propose the Medium class as suitable superclass, with one more ancestor (the Substance concept). Therefore we have DoI=1 for UMD and DoI=2 for SWEET. 


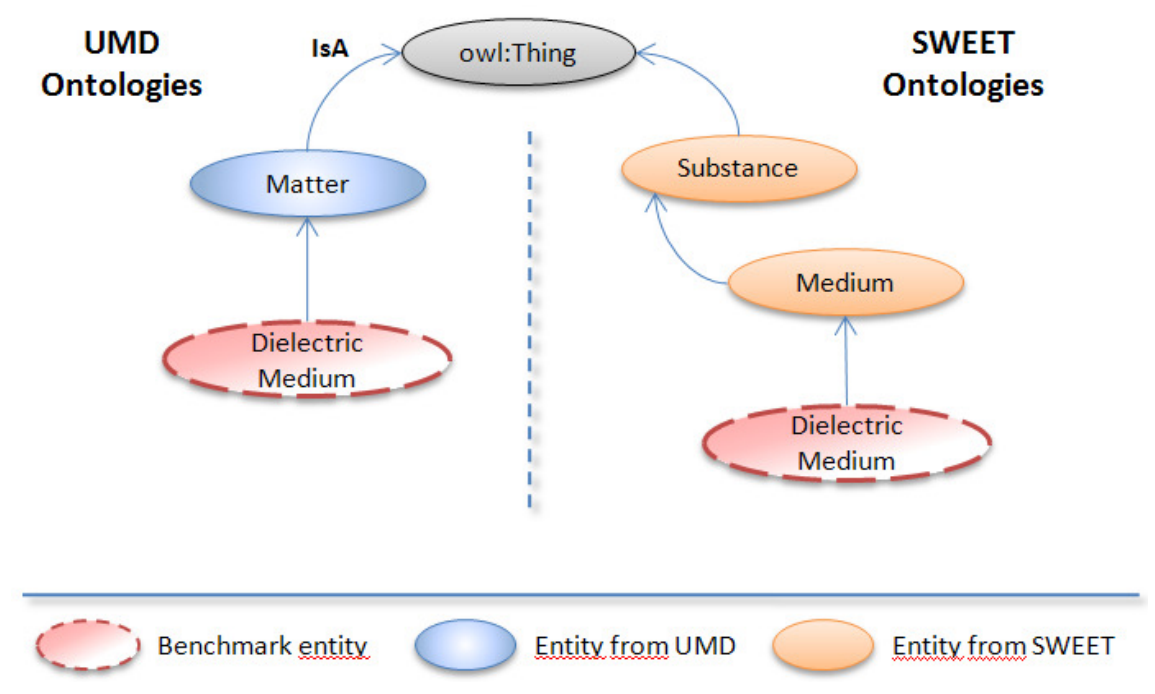

Figure 3. "Dielectric Medium” benchmark entity

As for the domain adequacy, both the ancestors in SWEET satisfy this requirement, therefore $I M=1$. UMD superclass is too general to be a useful direct superclass for Dielectric Medium and was not counted as suitable ancestor, resulting in $I M=0$.

We set $D o I_{r e f}=3$ as a valid depth of inheritance reference value for the EM knowledge domain, therefore we have $I D=0.33$ for UMD and $I D=0.67$ for SWEET.

Then we have to choose a descriptive statement in order to compute the $D P R$ metric for the benchmark entity. We considered the following: "a dielectric medium has insulation properties and can be polarized by an Electric field. It has some important key properties such as relative permittivity $\left(\varepsilon_{r}\right)$, quality factor $(Q)$ and measurement frequency”. As described in subsection 5.3, the statement is partitioned in a set of six natural language restrictions $(n N L R=6)$.

1) "to have insulation properties";

2) "to be polarized" or "to have polarizability";

3) "to be affected by an electric field";

4) "to have relative permittivity as a parameter";

5) "to have quality factor as a parameter";

6) "to have a measurement frequency as reference".

In UMD they become:

1) "hasquantity SOME RelativePermittivity";

2) "hasQuantity SOME QFactor";

where hasQuantity is the reusable property $(n R P=1$ and $D P R=0.17)$.

In SWEET they can be rendered as:

1) "hasRole ONLY Insulator";

2) "hasprocess SOME Polarization";

3) "hasParameter SOME RelativePermittivity"; 
4) "hasparameter SOME QFactor";

5) "hasfrequency SOME MeasurementFrequency";

where hasRole, hasprocess, hasParameter and hasfrequency are the reusable properties $(n R P=4$ and $D P R=0.67)$.

Table 6 summarizes integration-related metric computation for this benchmark entity.

Table 6. Integration metrics for "Dielectric Medium" benchmark entity

\begin{tabular}{|c|c|c|}
\hline Metric & UMD & SWEET \\
\hline IM & 0 & 1 \\
\hline ID & 0.33 & 0.67 \\
\hline DPR & 0.17 & 0.67 \\
\hline
\end{tabular}

\subsection{Metric overview}

The metrics proposed in the previous section consider many heterogeneous ontological aspects. Moreover, as they are all closed-ended and share the same numerical definition range, they can expose different behaviors at the endpoints according to the quantities they respectively monitor. Finally, those metrics can be classified not only on the basis of their group membership (size, content or integration) but also with respect to other features:

- to be dependent on the OWL codification language syntax;

- to be objective or subjective (i.e. to be computable automatically or not);

- to require preliminary setup or not (for instance: size and content metrics can be applied to the candidates and computed immediately whilst integration metrics need a simulation of the integration scenario as they refer to benchmark entities).

Therefore, Table 7 summarizes all the metrics and the above listed characteristics.

Table 7. Metric summary

\begin{tabular}{|c|c|c|c|c|}
\hline \multirow{2}{*}{ Metric } & \multirow{2}{*}{ Type } & \multirow{2}{*}{ Monitored quantity } & \multicolumn{2}{|c|}{ Value at endpoints } \\
\hline & & & $\approx 0$ & $\approx 1$ \\
\hline CtEr & $\begin{array}{c}\text { Objective } \\
\text { OWL-related }\end{array}$ & OWL Classes & Low & High \\
\hline PtEr & $\begin{array}{c}\text { Objective } \\
\text { OWL-related }\end{array}$ & $\begin{array}{l}\text { OWL Object Properties } \\
\text { OWL Datatype Properties }\end{array}$ & Low & High \\
\hline ItEr & $\begin{array}{c}\text { Objective } \\
\text { OWL-related }\end{array}$ & OWL Individuals & Low & High \\
\hline EpMr & $\begin{array}{c}\text { Objective } \\
\text { OWL-related } \\
\end{array}$ & OWL Entities per module & High & Low \\
\hline DSR & Subjective & Scientific Domain Entities & Low & High \\
\hline
\end{tabular}


International Journal of Web \& Semantic Technology (IJWesT) Vol.2, No.3, July 2011

\begin{tabular}{|c|c|c|c|c|}
\hline DER & Subjective & EM Domain Entities & High & Low \\
\hline LO & Subjective & Unused entities & High & Low \\
\hline \hline IM & $\begin{array}{c}\text { Subjective } \\
\text { OWL-related } \\
\text { Preliminary setup }\end{array}$ & $\begin{array}{c}\text { Appropriate scientific } \\
\text { superclasses for EM } \\
\text { concepts }\end{array}$ & Low & High \\
\hline ID & $\begin{array}{c}\text { Subjective } \\
\text { OWL-related } \\
\text { Preliminary setup }\end{array}$ & $\begin{array}{c}\text { Deviation from the } \\
\text { reference value of } \\
\text { scientific superclasses for } \\
\text { EM concepts }\end{array}$ & Low & High \\
\hline DPR & $\begin{array}{c}\text { Subjective } \\
\text { OWL-related } \\
\text { Preliminary setup }\end{array}$ & $\begin{array}{c}\text { Probability of reuse of } \\
\text { scientific OWL properties }\end{array}$ & Low & High \\
\hline
\end{tabular}

\section{ONTOLOGY RANKING}

As multi-decisional scoring methodology we adopt the ELECTRE I method [19]. It belongs to the ELECTRE family (ELimination Et choix Traduisant la REalité, that stands for Elimination and Choice Expressing the Reality) [20] and is widely used for its simplicity and its ability to cope with criteria giving discordant evaluations.

Our metrics, called evaluation criteria $\left(\boldsymbol{C}=\left\{C_{i}\right\}, i=1, \ldots, m\right)$ according to ELECTRE terminology, are weighted by using subjective quantities $\left(\boldsymbol{W}=\left\{\mathrm{w}_{i}\right\}, i=1, \ldots, m\right)$. A decision table (Table 8) is populated by the scores $a_{i j}$, expressing the performance of the $A_{i}$ alternative against the $C_{j}$ criterium. The alternatives are compared, in pair, by calculating concordance $\left(c_{j k}\right)$ or discordance $\left(d_{j k}\right)$ indices [19] according to (14) formulas.

$$
\begin{gathered}
c_{j k}=\frac{\sum_{i \in\left(C_{j k}^{+} \cup C_{j k}^{=}\right)^{w_{i}}}}{\sum_{i \in C_{j k}} w_{i}}=\sum_{i \in\left(C_{j k}^{+} \cup C_{j k}^{=}\right)} w_{i}, \\
d_{j k}=\frac{\max _{i \in C_{j k}^{-}}\left\{w_{i}\left|a_{i j}-a_{i k}\right|\right\}}{\max _{i \in C}\left\{w_{i}\left|a_{i j}-a_{i k}\right|\right\}}, \\
\boldsymbol{C}_{j k}=\boldsymbol{C}_{j k}^{+} \cup \boldsymbol{C}_{j k}^{=} \cup \boldsymbol{C}_{j k}^{-}
\end{gathered}
$$

where $\boldsymbol{C}^{+}{ }_{j k}, \boldsymbol{C}^{=}{ }_{j k}$ and $\boldsymbol{C}_{j k}^{-}$represent respectively the subset of criteria against which alternative $A_{j}$ is

\begin{tabular}{|c|c|c|c|c|c|c|}
\hline \multirow[b]{2}{*}{ Criteria } & \multirow[b]{2}{*}{ Weights } & \multicolumn{4}{|c|}{ Alternatives } & \\
\hline & & $A_{l}$ & . & • & $\overline{A_{n}}$ & \\
\hline$C_{1}$ & $W_{1}$ & $a_{11}$ & . & . & $a_{l n}$ & $A_{l}$ \\
\hline . & . & $\cdot$ & . & . & . & $\cdot$ \\
\hline . & . & . & . & . & . & . \\
\hline$C_{m}$ & $w_{m}$ & $a_{m l}$ & . & . & $a_{m n}$ & $A_{n}$ \\
\hline
\end{tabular}
better, equivalent and worst than $A_{k}$.

Table 8. ELECTRE-I typical decision table 
In order to conclude that the alternative $A_{j}$ outranks $A_{k}$, the concordance index should be at the same time above a concordance threshold $c_{t h}$ and the discordance index should be below a discordance threshold $d_{t h}(15)$, where $c_{t h}$ and $d_{t h}$ are the mean values of the indices calculated over the $n$ alternatives [19].

$$
\left\{\begin{array}{l}
c_{j k} \geq c_{t h}=\frac{1}{n(n-1)} \sum_{j=1}^{n} \sum_{\substack{k=1, k \neq j}}^{n} c_{j k} \\
d_{j k} \leq d_{t h}=\frac{1}{n(n-1)} \sum_{j=1}^{n} \sum_{\substack{k=1, k \neq j}}^{n} c_{j k}
\end{array}\right.
$$

\subsection{Candidate Ontology Ranking}

In this section the application of the proposed ranking methodology to our candidates is discussed.

Firstly, metrics were weighted. We assumed [19] that the sum of the weights of all criteria equals to 1 .

As for size metrics (i.e. CtEr, PtEr, ItEr and EpMr) we chose to assign them the same weight. Therefore we have $w_{C t E r}=w_{P t E r}=w_{I t E r}=w_{E p M r}=0.05$.

As for content metrics (i.e. $D S R, D E R$ and $L O$ ), the first one is the most relevant criterion in our opinion. Indeed, the more $S$ entities there are, the more suitable to integration the scientific candidate ontology could be. In addition, $E$ entities introduce semantic overlaps which may render the integration task onerous, therefore the $D S R$ metric has the second heaviest weight. This determines the following weights: $w_{D S R}=0.2, w_{D E R}=0.15, w_{L O}=0.05$.

As to integration metrics, we assigned the heaviest weight to $I M$, as it expresses the scientific suitability of higher-level concepts. The availability of reusable properties is an important feature as well, therefore we assigned to $D P R$ the second heaviest weight in this subset. We have: $w_{I M}=0.2, w_{I D}=0.1, w_{D P R}=0.15$.

Table 9 resumes the final decision table. The highest evaluation scores for each alternative against the available criteria have been highlighted. $I M, I D$ and $D P R$ values refer to the mean over the totality of the benchmark entities.

Table 9. ELECTRE-I decision table for the selected scientific domain ontologies

\begin{tabular}{|c|c|c|c|}
\hline $\mathbf{C}_{\mathbf{i}}$ & $\mathbf{w}_{\mathbf{i}}$ & $\mathbf{A}_{\mathbf{1}}$ (UMD) & $\mathbf{A}_{\mathbf{2}}$ (SWEET) \\
\hline $\mathbf{C t E r}$ & 0.05 & 0.88 & 0.63 \\
\hline $\mathbf{P t E r}$ & 0.05 & 0.06 & 0.08 \\
\hline $\mathbf{I t E r}$ & 0.05 & 0.05 & 0.28 \\
\hline EpMr & 0.05 & 0.923 & 0.995 \\
\hline \hline DSR & 0.2 & 0.44 & 0.59 \\
\hline
\end{tabular}


International Journal of Web \& Semantic Technology (IJWesT) Vol.2, No.3, July 2011

\begin{tabular}{|c|c|c|c|}
\hline DER & 0.15 & 0.94 & 0.93 \\
\hline LO & 0.05 & 0.5 & 0.66 \\
\hline \hline IM & 0.2 & 0.667 & 0.91 \\
\hline ID & 0.1 & 0.398 & 0.659 \\
\hline DPR & 0.15 & 0.386 & 0.711 \\
\hline
\end{tabular}

The following matrices report the concordance and discordance indices of the alternatives.

$$
\begin{gathered}
C=\left[\begin{array}{cc}
\cdot & c_{12} \\
c_{21} & \cdot
\end{array}\right]=\left[\begin{array}{cc}
\cdot & 0.2 \\
0.8 & \cdot
\end{array}\right] \\
D=\left[\begin{array}{cc}
\cdot & d_{12} \\
d_{21} & \cdot
\end{array}\right]=\left[\begin{array}{cc}
\cdot & 1 \\
0.256 & \cdot
\end{array}\right]
\end{gathered}
$$

According to (14), we have the following concordance and discordance conditions against respective thresholds:

$$
\begin{gathered}
\left\{\begin{array}{c}
\left(c_{12}=0.2\right)<\left(c_{t h}=0.5\right) \\
\left(d_{12}=1\right)>\left(d_{t h}=0.628\right)
\end{array}\right. \\
\left\{\begin{array}{c}
\left(c_{21}=0.8\right)>\left(c_{t h}=0.5\right) \\
\left(d_{21}=0.256\right)<\left(d_{t h}=0.628\right)
\end{array}\right.
\end{gathered}
$$

As (19) satisfies both the conditions, the alternative $A_{2}$ (i.e. SWEET ontologies) shows a better integration behavior rather than $\mathrm{A}_{1}$ (i.e. the UMD ontologies). As a consequence, we can rigorously determine that $A_{2}$ outranks $A_{1}$.

These results confirmed the assessments given by independent EM knowledge domain experts who, based on a preliminary overview of candidate ontology contents and on the evaluation of their "electromagnetic soundness", accounted SWEET ontologies as the most profitable choice among candidates.

\section{Conclusions}

In this paper a comparison among two different scientific domain ontologies has been presented in order to determine which one would perform better if integrated as higher semantic layer with proprietary EM-domain ontologies defined in OntoCEM framework. A set of ad-hoc metrics was codified in order to evaluate different aspects of candidate ontologies: size and structure, contents, integration-related procedures. Metric computation takes into account the domain expert point of view and analyzes basic ontological design aspects. These metrics were used as ranking criteria in a multi-criteria decisional analysis based on the application of the ELECTRE I method. Results demonstrated that an unambiguous outranking relation among the all evaluated candidates can be obtained by applying the proposed methodology. Additional features such as the realization of an ontological editor plugin to classify domain ontologies entities according to the domain expert opinion are at a development stage. 
International Journal of Web \& Semantic Technology (IJWesT) Vol.2, No.3, July 2011

\section{REFERENCES}

[1] Gruber, T. (1995), "Towards Principles for the Design of Ontologies Used for Knowledge Sharing", in: Int. Journal of Human-Computer Studies, Vol.43, Issues 5-6, November 1995, pp.907-928.

[2] Beale, T. \& Heard, S. (2007), “An Ontology-based Model of Clinical Information”, in: Proc. of MedInfo 2007, K. Kuhn et al. (Eds), pp.760-764, IOS Publishing.

[3] OpenClinical Ontologies homepage, http://www.openclinical.org/ontologies.html (last accessed: May 2011).

[4] Lesteven S., et al. (2006), "Ontologies For Astronomy", in: Library and Information Services in Astronomy V: Common Challenges, Uncommon Solutions. ASP Conference Series, vol.377, p.193.

[5] Mastella, L et al. (2008), "Ontology-based Model Annotation of Heterogeneous Geological Representations", in: Proc. of the $4^{\text {th }}$ Int. Conf. on Web Information Systems and Technologies (WEBIST08), Madeira, Portugal.

[6] Web Ontology Language For Astronomy, "Astronomy/Science Ontology", Department of Astronomy, University of Maryland, USA. Available at: http://www.astro.umd.edu/ eshaya/ (last accessed: May 2011).

[7] Semantic Web for Earth and Environmental Terminology (SWEET) Ontologies, Jet Propulsion Laboratory, California Institute of Technology Available at: http://sweet.jpl.nasa.gov/ (last accessed: May 2011).

[8] Davenport, J.H. \& Kohlhase, M. (2009), "Unifying Math Ontologies: A Tale of Two Standards", in: Intelligent Computer Mathematics, Lecture Notes in Computer Science, vol.5625/2009, pp.263278, DOI: 10.1007/978-3-642-02614-0_23.

[9] OPB, Ontology of Physics for Biology, Biomedical and Health Informatics, University of Washington. Homepage: http://www.bhi.washington.edu/research/SemBioProcess/OPB.htm (last accessed: May 2011).

[10] Durand, N. et al. (2007), "Ontology-Based Object Recognition for Remote Sensing Image Interpretation", in: Proc. of the $19^{\text {th }}$ IEEE Int. Conf. on Tools with Artificial Intelligence (ICTAI2007), vol.1, pp.472-479.

[11] Esposito, A., Tarricone, L., Vallone, L. \& Zappatore, M. (2008), "A Proposal for an Electromagnetic Ontology Framework", in: Int. Journal of Web and Grid Services, Vol.4, No.3, pp.284-302.

[12] Pinto, H.S. (1999), “Towards Ontology Reuse”, in: Proc. of AAAI99's Workshop on Ontology Management, pages 67-73. AAAI Press.

[13] Pinto, H.S. \& Martins, J.P. (2001), "A methodology for ontology integration", in: Proc. of the 1st Int. Conf. on Knowledge Capture, October 22-23, 2001, Victoria, British Columbia, Canada.

[14] W3C OWL Working Group, “OWL 2 Web Ontology Language Document Overview”, W3C Recommendation, 27th October 2009. Available at: http://www.w3.org/TR/owl2-overview/ (last accessed: May 2011).

[15] Ontological Codification of ElectroMagnetism v.2.1 (OntoCEM), University of Salento, Electromagnetic Lab Lecce $\left(\mathrm{EML}^{2}\right)$ Progressively available at http://www.electromagnetics.unisalento.it/ontologies/OntoCEM/2.1/.

[16] Lethbridge, T. (1998), "Metrics for Concept-Oriented Knowledge Bases", in: Int. Journal of Software Engineering and Knowledge Engineering, vol.8(2), pp.161-188.

[17] Zhang, H. et al. (2010), "Measuring Design Complexity of Semantic Web Ontologies", in: The Journal of Systems and Software, Science Direct, vol.83, pp.803-814.

[18] Kalfoglou, Y. et al. (2006), "Semantic Metrics", in: Proc. of 5th Int. Conf. on Knowledge Engineering and Knowledge Management (EKAW06), pp.166-181. 
International Journal of Web \& Semantic Technology (IJWesT) Vol.2, No.3, July 2011

[19] Figueira, J. et al. (2005), "Multiple Criteria Decision Analysis: The State of the Art Surveys", Springer Science and Business Media, Inc., New York.

[20] Roy, B. (1968), "Classement et choix en présence de points de vue multiples (la méthode ELECTRE), in: RIRO (Revue d'Informatique et de Recherche Opérationnelle), 8:57-75.

[21] Web Ontology Language For Astronomy, "Astronomy/Science Ontology", Department of Astronomy, University of Maryland, USA. Available at: http://www.astro.umd.edu/ eshaya/ (last accessed: May 2011).

[22] Semantic Web for Earth and Environmental Terminology (SWEET) Ontologies, Jet Propulsion Laboratory, California Institute of Technology Available at: http://sweet.jpl.nasa.gov/ (last accessed: May 2011).

[23] International Virtual Observatory Alliance homepage. Available at: http://www.ivoa.net/ (last accessed: May 2011).

[24] Tartir, S. et al. (2005), "OntoQA: Metric-Based Ontology Quality Analysis", in: Proc. of IEEE Workshop on Knowledge Acquisition from Distributed, Autonomous, Semantically Heterogeneous Data and Knowledge Sources, Houston, Texas, USA, November.

[25] Guarino, N. (1998), "Formal ontology and information systems", in: N. Guarino (Ed.) Proc. of the 1st Int. Conf. on Formal Ontologies in Information Systems, FOIS'98, Trento, Italy: IOS Press, June 1998, pp.3-15.

[26] International Virtual Observatory Alliance homepage. Available at: http://www.ivoa.net/ (last accessed: May 2011).

[27] Raskin, R. (2006), "Guide to SWEET Ontologies", NASA/Jet Propulsion Lab, Pasadena,CA,USA, Available at: http://sweet.jpl.nasa.gov/guide.doc (last accessed: May 2011).

[28] Cheng, D.K. (1993), Fundamentals of Engineering Electromagnetics, Addison-Wesley Series in Electrical Engineering.

[29] M. Klein (2001), "Combining and Relating Ontologies: Problems and Solutions", in: Proc. of IJCAI Workshop on Ontologies, Seattle.

[30] H.S.Pinto \& J.P. Martins (2001), "A methodology for ontology integration", in: Proc. of the 1st Int. Conf. on Knowledge Capture, October 22-23, 2001, Victoria, British Columbia, Canada.

[31] S. Staab \& R. Studer Editors (2009), "Handbook on Ontologies - 2nd Edition", International Handbooks on Information Systems, Springer.

[32] R. Raskin (2006), "Guide to SWEET Ontologies", NASA/Jet Propulsion Lab, Pasadena,CA,USA,2006. Available at: http://sweet.jpl.nasa.gov/guide.doc (last accessed: May 2011).

[33] WordNet homepage. Available at: http://wordnet.princeton.edu/ (last accessed: May 2011).

[34] J.A. Kwak and H.S. Yong, "Ontology Matching Based on hypernym, hyponym, holonym, and meronym Sets in WordNet", in: International Journal of Web and Semantic Technology (IJWesT), Vol. 1, No. 2, pp. 1-14, April 2010.

[35] D. Beneventano, S. Bergamaschi, F. Guerra and M. Vincini, "Synthesizing an Integrated Ontology”, in: IEEE Internet Computing 7(5): 42-51, 2003. 
International Journal of Web \& Semantic Technology (IJWesT) Vol.2, No.3, July 2011

\title{
Authors
}

Luciano Tarricone is a Full Professor of Electromagnetic Fields at the University of Lecce, Italy. He received his laurea degree (with honors) from the University of Rome "La Sapienza", Italy, and his PhD from the same university, both in Electronic Engineering. In 1990 he was a researcher at the Italian National Institute of Health, and between 1990 and 1994 at the IBM European Center for Scientific and Engineering Computing. Between 1994 and 2001 he was at the University of Perugia, Italy. Since 2001 he has joined the University of Lecce. His main research areas are: supercomputing and knowledge engineering for Electromagnetics, environmental electromagnetic compatibility, CAD of microwave circuits and antennas. He authored around 300 papers in international conferences and journals, and edited 3 volumes in the area of high performance computing and knowledge engineering for electromagnetics.

\begin{abstract}
Alessandra Esposito is a free-lance consultant in the area of Computer Science and Information Technologies, with a focus on networking, web and grid applications for research in universities and small, medium and large companies. She received her laurea degree (with honors) in Electronic Engineering from the University of Naples. Between 1990 and 1994 she was with IBM Scientific Center in Rome, Italy. In 1994 and 1995 she was a system engineer for Sodalia, Trento, Italy, involved in research and development in the area of distributed systems. Since 1995 she has cooperated with several research institutions, universities and business companies, in the framework of educational, research and industrial projects. She authored about 90 papers in international conferences and journals. She is co-author of the book "Grid Computing for Electromagnetics" edited by da Artech House.
\end{abstract}

Marco Zappatore is a PhD Student in Information Engineering at the University of Lecce. He graduated in Information Engineering at the same University in 2005 with a thesis dealing with an hybrid genetic algorithm for 3G wireless network optimum planning. In 2008, He received a Laurea Specialistica degree (2nd level) in Telecommunications Engineering from the same University with a thesis concerning the EM enabling technologies for software intelligent systems in the healthcare domain. He currently collaborates with the Electromagnetic Fields Group at University of Salento. His research activities are mainly focused on: Semantic Web, Context-Awareness and Multi-Agent Systems applied to the EM area, optimization techniques for wireless networks planning. He co-authored about 20 papers in international conferences and journals. 